\title{
Xom as a novel partner of Lef/Tcfs during dorsal-ventral patterning of the Xenopus embryo
}

\author{
Yingzi Yang $^{1}$ \\ ${ }^{1}$ Genetic Disease Research Branch, National Human Genome Research Institute, Bethesda, MD 20892, USA
}

Cell Research (2007) 17:307-308. doi: 10.1038/cr.2007.29; published online 16 April 2007

Both the Wnt/ $\beta$-catenin and Bone morphogenetic protein (Bmp) signaling pathways play critical roles in dorsal-ventral patterning of the Xenopus embryos. The lymphoid enhancer binding factor (Lef) / T cell factor (Tcf) have been viewed as dedicated transcription factors of the $\mathrm{Wnt} / \beta$-catenin signaling pathway that are activated by $\beta$-catenin binding. Now a Xenopus ventral specific transcription factor Xom has also been identified to bear transactivation activity by binding to Lef/Tcf factors [1].

One of the earliest events in vertebrate development is the establishment of body axes, which are anterior-posterior (A-P, from head to tail), dorsal-ventral (D-V, from the backbone to the belly) and left-right (i.e., the heart loops from left to right). Dorsal and ventral cells give rise to distinct tissues later in development. For instance, dorsal ectoderm gives rise to neural plate which will form the entire central nervous system while ventral ectoderm forms epidermis. Classic embryological manipulations of Xenopus embryos by Spemann and Mangold [2] have established that secreted signals from a dorsal blastopore lip (the dorsal organizer, equivalent to the shield in zebrafish, node in mouse, and Henson's node in chick) can re-specify ventral tissues to become those from the dorsal origin. Molecular embryological and genetic studies in Xenopus and zebrafish (whose early embryonic development shares extensive similarities with that in the Xenopus) have led to the conclusion that specification of ventral fate also relies on a functional ventral cell signaling center. While the Wnt/ $\beta$-catenin pathway establishes the location of the dorsal most aspect of the $\mathrm{D}-\mathrm{V}$ axis, referred as the Spemann organizer, Bmp signaling in the ventral side is both necessary and sufficient for ventral fate determination. There is a mutual antagonism between the dorsal and ventral signals. The $\mathrm{Wnt} / \beta$-Catenin signaling in the Spemann organizer induces the expression of Bmp antagonist genes such as Noggin (Nog) and Chordin (Chd) in dorsal regions, thus initiating the restriction of Bmp signaling ventrolaterally. On the other hand, Bmp signaling in the ventral side promotes expression of Bmp ligands and the Vent family transcription factors (i.e., Xom) that repress dorsal gene expression in ventral regions (reviewed by De Robertis and Kuroda, 2004) [3]. The proper regulation of the mutual antagonism between dorsal and ventral signaling is essential for the initial establishment of the dorsal and ventral domains in the early Xenopus embryo.

The paper by Gao et al. [1], in this issue of Cell Research uncovered a novel aspect of Xom function in mediating the cooperative, but not antagonistic interaction of the $\mathrm{Wnt} / \beta$-catenin and Bmp signaling during $\mathrm{D}-\mathrm{V}$ patterning of the Xenopus embryo. It is found in the paper that besides repressing dorsal gene expression, Xom can also act as a transcription activator by directly binding Lef/Tcfs and transactivating the Lef/Tcf-mediated transcription of ventral specific genes through its N-terminal transactivation domain (TAD). The Lef/Tcf factors are a family of high mobility group (HMG) transcription factors, whose activities depend on Lef/Tcf associated factors. The Lef/Tcf factors have been viewed as dedicated transcription factors of the Wnt/ $\beta$-catenin signaling pathway. In the absence of Wnt ligand, the Lef/Tcfs are associated with transcription repressors, such as Grouch and CtBP, which maintain the Lef/Tcf-mediated transcription in a repressed state. Upon Wnt signaling, the most significant event is the inhibition of $\beta$-catenin degradation such that stabilized $\beta$-catenin translocates to the nucleus where it binds Lef/Tcfs and activates downstream gene expression (reviewed in Logan and Nusse, 2004) [4]. Interestingly, the same Wnt/ $\beta$-catenin signaling pathway acts at two differ- 
ent stages to control D-V patterning in early Xenopus embryos. First, Wnt signaling components encoded by maternal mRNA establish the D-V embryonic axis by promoting the nuclear localization of $\beta$-catenin specifically on the dorsal side of the embryo. Second, zygotically expressed Xwnt-8 promotes ventral and lateral and restricts dorsal mesodermal development. This second activity is almost the opposite of the first one. Indeed, Lef/Tcf binding sequences are found in promoters of ventral-specific genes including Xom itself and loss of Lef1 activity in Xenopus leads to ventral, rather than dorsal defects [5]. In zebrafish embryos, it has been shown that Xom expression is under both Wnt8 and Bmp control, but that Wnt8 is the primary regulator [6].

Although it still awaits further confirmation by the chromatin immunoprecipitation (CHIP) assays using the ventral cells from the early Xenopus embryos, the discovery that Xom can activate Lef/Tcf-mediated transcription indicates that $\mathrm{Wnt} / \beta$-catenin is not the only signaling pathway that can regulate ventral gene expression through Lef/Tcfs in the Xenopus embryo. In support of this, it has been observed in zebrafish embryos that over-expression of a conditional dominant repressor form of Tcf (hs- $\Delta \mathrm{Tcf}$ ) leads to a more severe phenotype than the loss of Wnt8 [7]. In addition, after initial activation by Xwnt8, Xom may maintain its own expression and thus the ventral cell fates by binding Lef/Tcfs on its own promoter independently of the $\mathrm{Wnt} / \beta$-catenin signaling. Lef/Tcf controlled $\beta$-galactosidase expression in the Topgal mice has been used as an in vivo readout for the Wnt/ $\beta$-catenin signaling activation [8]. If Lef/Tcf-mediated transcription can be activated by factors other than $\beta$-catenin, one has to take special caution when the Wnt/ $\beta$-catenin signaling activity is assessed by examining the $\beta$-galactosidase expression in the Topgal mice.

Since activation of Lef/Tcf-mediated transcription by $\beta$-catenin has been implicated in the proliferation of stem cells and the initial step of malignant tumor formation $[9,10]$, it is possible that factors not in the Wnt/ $\beta$-catenin signaling pathway, such as Xom, may also regulate stem cell proliferation and differentiation through binding Lef/Tcf factors. In addition, abnormal expression of Xom may be found to cause tumors through activating Lef/Tcf-mediated transcription. However, there might be both qualitative and quantitative differences between $\beta$-catenin-activated and Xom-activated Lef/Tcf-mediated transcription and such differences may be determined by the additional components in the Lef/Tcf transcriptional complex.

\section{References}

1 Gao H, Wu B, Giese R, Zhu Z. Xom interacts with and stimulates transcriptional activity of LEF1/TCFs: implications for ventral cell fate determination during vertebrate embryogenesis. Cell Res 2007; 17:345-356.

2 Spemann H, Mangold H. Induction of embryonic primordia by implantation of organizers from a different species. 1923. Int J Dev Biol 1924; 45:13-38.

3 De Robertis EM, Kuroda H. Dorsal-ventral patterning and neural induction in Xenopus embryos. Annu Rev Cell Dev Biol 2004; 20:285308.

4 Logan CY, Nusse R. The wnt signaling pathway in development and disease. Annu Rev Cell Dev Biol 2004; 20:781-810.

5 Roel G, Hamilton FS, Gent Y, Bain AA, Destree O, Hoppler S. Lef-1 and Tcf-3 transcription factors mediate tissue-specific Wnt signaling during Xenopus development. Curr Biol 2002; 12:1941-1945.

6 Ramel MC, Lekven AC. Repression of the vertebrate organizer by Wnt8 is mediated by Vent and Vox. Development 2004; 131:39914000.

7 Lewis JL, Bonner J, Modrell M, et al. Reiterated Wnt signaling during zebrafish neural crest development. Development 2004; 131:12991308.

8 DasGupta R, Fuchs E. Multiple roles for activated LEF/TCF transcription complexes during hair follicle development and differentiation. Development 1999; 126:4557-4568.

9 Korinek V, Barker N, Moerer P, et al. Depletion of epithelial stem-cell compartments in the small intestine of mice lacking Tcf-4. Nat Genet 1998; 19:379-383.

10 Korinek V, Barker N, Morin PJ, et al. Constitutive transcriptional activation by a beta-catenin-Tcf complex in APC-/- colon carcinoma. Science 1997; 275:1784-1787. 